\title{
Perioperative Predictors of Ultra-Fast-Track Failure and Prolonged Hospital Length of Stay in a Preliminary Protocol of Enhanced Recovery after off-Pump Coronary Artery Bypass Grafting
}

\author{
Gustavo Muñoz-Galarce, MD ${ }^{1}$, Gustavo Muñoz-Monaco, MD², Máximo C. Guida, MD ${ }^{3}$ \\ Nadine Odo, BA, ELS ${ }^{4}$, Carlos Ramirez-Paesano, MD*5 \\ ${ }^{1}$ Adult Cardiac Anesthesiologist, FundaCardio Foundation, Valencia, Venezuela. \\ ${ }^{2}$ Resident Physician.Pediatric Cardiac Anesthesiology, Pediatric Anesthesiology Fellow, Medical College of \\ Wisconsin at Children's Hospital of Milwaukee, USA. \\ ${ }^{3}$ Adult Cardiac Surgeon, FundaCardio Foundation, Valencia, Venezuela. \\ ${ }^{4}$ Research Associate, Anesthesiology and Perioperative Medicine, Medical College of Georgia, Georgia, USA. \\ ${ }^{5}$ Adult Cardiac Anesthesiologist, Anesthesiology Service (Anestalia).Centro MédicoTeknon, Spain. \\ carrampa@hotmail.com
}

*Corresponding Author: Carlos Ramirez-Paesano, MD, Anesthesiology Service (Anestalia). Centro MédicoTeknon, calle Vilana 12, Barcelona, 08022, Spain.

\section{Abstract}

Background: The use of Ultra-fast-track (UFT) management in cardiac surgery may shorten hospital length of stay (Hosp-LOS) when is part of a protocol of enhance recovery after surgery (ERAS).

Methods: We retrospectively analyzed the data of the patients undergoing elective off-pump coronary artery bypass grafting (OPCAB) using UFT under a pilot program of ERAS at a Venezuelan nonprofit cardiac center from 2010 to 2014. The primary goal was to describe the short-term outcome of a consecutive case-series managed with desflurane-remifentanil-intercostals nerve block for OPCAB by Left-Anterolateral Thoracotomy and followed up by multidisciplinary enhance recovery pathway. The secondary goals were to identify perioperative predictors for UTF-failure and Hosp-LOS> 4 days.

Results: 1,943 bypasses were performed on 673patients. $61.5 \pm 9.5$ years old, EuroSCORE was $5.2 \pm 4.1$. $97.8 \%$ was extubated in Operating Room (UFT-success) and $2.2 \%$ extubated in the intensive care unit (UFT-failure). The reintubation rate was 0.5\%. Patients had an Intensive Care Unit length of stay (ICU-LOS) of $29 \pm 4.2$ hours; 636 patients (94.5\%) had ICU-LOS $\leq 24$ hrs, 2.1\% readmitted to ICU. The Hosp-LOS after discharge from ICU was $50.5 \pm 9.9$ hours, 633 (94.1\%) had Hosp-LOS $\leq 4$ days. Univariate analysis revealed as independent risk factors for UFT-failure: age, female sex, EuroSCORE, Severity of Angina Pectoris, EF $<30 \%$, Redo, COPD, PRBC transfusion, use of elective IABP and duration of surgery ( $t-Q x)$. However, multivariate logistic regression analysis and backward elimination method found as strong risk factors for UFT-failure: transfusion of $\geq 2 P R C B$ Adjusted Odds Ratio (AOR=6.02) (95\%CI) ( $p<0.05), t-Q x 3-4 \mathrm{hrs,}(A O R=77.3)(95 \% C I)(p<0.001)$ and $t-Q x>4$ hrs, $(A O R=157.5)(95 \% C I)(p<0.001)$. Univariate analysis revealed as independent risk factors for Hosp-LOS $>4$ days: Age $>80$ yo ,female sex, NYHA $>I I I$, EuroSCORE, severity of Angina pectoris, EF $<30 \%$, Redo, renal failure, $I A B P, P R C B$ transfusion, UFT-failure, $t-Q x$ and ICU-LOS ( $p<0.001)$. Multivariate logistic regression analysis and backward elimination method found as strong risk factors for Prolonged Hosp-LOS ( $>4$ days): Redo (AOR=7.68) (95\%CI), $t-Q x>3$ hrs $(A O R>74)(95 \% C I)(p<0.001)$ and ICU-LOS $>24 h r s(A O R=29.3)(95 \% C I)(p<0.001)$. 
Perioperative Predictors of Ultra-Fast-Track Failure and Prolonged Hospital Length of Stay in a Preliminary Protocol of Enhanced Recovery after off-Pump Coronary Artery Bypass Grafting

Conclusions: As most patients were extubated in the Op.Room, had short ICU-LOS ( $<24 \mathrm{hrs}$ ) and short HospitalLOS ( $\leq 4$ days). UTF appears to have clinical benefits in this setting. In general, age $>80$ y.o, female sex, severity of EUrOSCORE and $\geq 2$ PRBCs transfusion were risk factor for UTF-failure and prolonged Hosp-LOS. However, the stronger predictive factors for UTF-failure and Hosp-LOS $>4$ days were transfusion of $\geq 2$ PRCB, Redo, duration of surgery $>3$ hrs and ICU-LOS $>24 \mathrm{hrs}$. Prospective studies would better elucidate the risk factors for longer Hospital-LOS and attendant morbidities.

Keywords: Ultra-fast track, off-pump coronary artery bypass graft, left anterolateral thoracotomy, beating heart, early extubation, enhanced recovery after surgery (ERAS).

\section{INTRODUCTION}

Fast track anesthesia in cardiac surgery, which enables extubation within 0-6 hours of the end of surgery, gained acceptance after the 1990s when it was proven to reduce intensive care unit length of stay (ICU-LOS), use of resources, and cost. ${ }^{1}$ The same could be said for ultra-fast track (UFT) anesthesia with the use off-pump coronary artery bypass grafting (OPCAB) which enables extubation in the operating room (Op.Room), immediately after the surgery is completed, with relatively few major complications. ${ }^{2-5}$ A direct relationship has been suggested between UFT extubation and reductions in the use of sedatives, analgesics, intravenous fluids, inotropic agents, vasopressors, and anti-arrhythmics in the ICU. ${ }^{6,7}$ Originally designed for low-risk patients, immediate extubation after OPCAB is becoming more common for high-risk cardiac surgery patients. ${ }^{8}$ OPCAB procedures managed with fast-track protocols have been carried out by median sternotomy, ${ }^{9}$ by left anterolateral thoracotomy (LAT), ${ }^{10}$ and with minimally invasive direct coronary artery bypass (MIDCAB), with or without staged hybrid revascularization..$^{11,12}$

Enhanced recovery pathways are multimodal, evidencebased protocols including step-by-step management plans throughout the perioperative period. The benefits of UFT anesthetic management after OPCAB should be supported by structured protocols of enhance recovery after surgery (ERAS) properly adapted to this type of surgery and also to each hospital. Evidences suggest that enhanced recovery pathways improve postoperative recovery, reduce morbidity and hospital length of stay(Hosp-LOS), and cost of care for variety of surgical procedures by mean of attenuating perioperative physiologic stress (eg. psychological support, preoperative oral carbohydrate, respiratory exercise training, and afferent neural blockade) and decreasing the use of interventions that slow down recovery progress without improving outcomes (eg. prolonged preoperative fasting, prolonged urinary drains, delayed postoperative feeding $)^{13}$. Majority of ERAS evidence come from abdominal surgery ${ }^{14}$, and some reports describe the impact of UFT plus ERAS on outcomes of OPCAB and thoracic surgery ${ }^{15}$.

The aims of this descriptive and retrospective study were: 1 ) to show the short- term outcome ( $\leq 30$ days) of a consecutive case series of 673 patients managed by UFT anesthetic technique utilizing a combination of desflurane, remifentanil, and intercostals nerve block in OPCAB by the LAT approach and supported by multidisciplinary enhance recovery pathway; 2 ) to identify perioperative predictors for UTF-failure and Hosp-LOS> 4 days.

\section{METHODS}

With institutional review board approval, we collected retrospective data of consecutive patients who had undergone OPCAB between June 2010 and December 2014 and met the criteria for extubationin-Op.Room and followed up by an adapted ERAS protocol at a nonprofit cardiovascular surgery center (FundaCardio Foundation, Valencia, Venezuela). Emergencies, and pediatric procedures were excluded. The ethics committee of the hospital provided us with a coded database for the retrospective review following strict compliance with the regulations of personal data protection.

Data collected included patient demographics and comorbidities, European System for Cardiac Operative Risk Evaluation (EuroSCORE) and New York Heart Association (NYHA) functional class, whether patients were extubated in the Op.Room(UFT-success) or ICU (UFT-failure), intraoperative and postoperative complications, ICU-LOS, Hosp-LOS, and mortality at 30 days. 
Perioperative Predictors of Ultra-Fast-Track Failure and Prolonged Hospital Length of Stay in a Preliminary Protocol of Enhanced Recovery after off-Pump Coronary Artery Bypass Grafting

\section{Enhanced Recovery after Surgery (ERAS)}

Since the end of 2009 year, our team began to implement and testing a preliminary multidisciplinary program of structured perioperative support to provide early recovery after OPCAB in elective patients as complement of UTF protocol. The objective of the early recovery pathway was to introduce a standard multimodal approach, including preoperative patient and family education, psychological and nutrition support, opiod-sparing pain management, early and conducted mobilization, early feeding as possible and standardized drains management. Elements of the early recovery pathway used a specific physician and surgeon orders, patients-adapted cardiologic orders, daily nursing records and patient education booklet. The team was led by a surgeon (full-time coordinator) and an anesthesiologist, also included a cardiologist, ICU physician, expertise in nursing(inpatient and outpatient), a full-time outpatient nursing coordinator, physiotherapy, pharmacy, pain service, nutrition services. Protocol is summarize in Appendix 1.

\section{Anesthesia Protocol}

The preanesthetic evaluation was scheduled after two previous ERAS-training interviews, as minimum. During the pre-anesthetic interview, we reviewed the patient's medical records, identify co-morbidities and chronic medications, evaluate anesthetic risk, and stratify by EuroSCORE and NYHA functional class, and perform a physical exam. If it necessary, call internist, cardiologist or pulmonologist to optimized any preexisting co-morbidity. Ask if the patients and family were properly informed and trained by ERAS team and check any doubt about perioperative information booklet. Then, we ask for the informed consent.

Prior to the surgery, routine medications are suspended as follows: Clopidogrel, prasugrel, or ticagrelor are suspended 7 days before surgery; dabigatran, rivaroxaban or apixaban, 5 days before surgery; aspirin, the day before surgery; and eptifibatide or tirofiban, 12 hours before surgery. Warfarin is suspended 5 days before surgery and the international normalized ratio is monitored until the target of $\leq 1.5$ is reached. Enoxiparine or another low molecular weight heparin is used when an anti- aggregant, anti-platelet, or anticoagulant is suspended; the last heparin dose is administered 24 hours before surgery. Pre-medication consists of oral benzodiazepine, midazolam $7.5 \mathrm{mg}$ the night before surgery with $100-150 \mathrm{ml}$ of Ensure
Clear ${ }^{\circledR}$ (Abbott Nutrition Products) a fat-free flavored nutrition clear liquid after dinner(regular diet until $9 \mathrm{pm}$ for the morning schedule patients, and $6 \mathrm{am}$ for afternoon schedule patients), and gabapentin $600 \mathrm{mg}$ two hours before surgery with $100 \mathrm{ml}$ of G2 Gatorade $₫($ PepsiCo) or Powerade $₫($ Coca-ColaCo.).

The goal of the anesthetic plan was immediate extubation in the Op.Room. On arriving in the Op.Room, a peripheral intravenous (IV) line is catheterized in the right upper limb, standard ASA monitoring is started, and an arterial line is catheterized into the right radial artery for invasive blood pressure (IBP) monitoring. After facemask oxygenation with a $\mathrm{FIO}_{2}$ of $100 \%$ for 5 minutes, induction of anesthesia is initiated with remifentanil at $0.15-0.2 \mu \mathrm{g} / \mathrm{kg} /$ min IV infused by a volumetric pump and titration of propofol at 1-1.5 mg/kg IV. When eyelash loss reflex is achieved and bispectral index is about 5055 , rocuronium bromide doses are administered at $0.6 \mathrm{mg} / \mathrm{kg} \mathrm{IV}$, and the patient is intubated when one response or less to train of four (TOF) is achieved. Trans-esophageal echocardiography (TEE) is then used to assess standard ASA views and identify any new feature or cardiac issue. The left subclavian vein is catheterized and central venous pressure is checked. The procedure then moves to the left pulmonary exclusion with a bronchial blocker guided by a flexible fiberoptic under $90 \%$ oxygen to manage the patient with right-sided one lung ventilation (OLV) to facilitate the surgical approach. Ventilation monitoring includes basic parameters of mechanical ventilation and pressure-volume and flow-volume loops. If selective ventilation cannot be established or the patient cannot tolerate OLV, the left lung is gently compressed with a laparotomy sponge. Maintenance of anesthesia is accomplished with desflurane 0.5-0.8 MAC $\left(\mathrm{ET}_{\left.\text {vol\% }_{0}\right)}\right.$ to maintain the bispectral index between 40 and 60 , a variable dose of remifentanil infusion to maintain hemodynamic stability (IBP and PR \pm 10 $15 \%$ of pre-anesthesia induction value), and ketamine $0.15 \mathrm{mg} / \mathrm{kg} / \mathrm{h} \mathrm{IV}$. Muscle relaxation (by monitoring post-tetanic count stimulation) is maintained at a profound to intense level with additional rocuronium doses at $0.3 \mathrm{mg} / \mathrm{kg}$ IV. For adequate control of body temperature, we use an underbody thermal blanket, administer normothermic endovenous fluids using a fluid warmer, and turn off air-conditioning in the surgical room. Corporal temperature is measured by a continuous nasopharyngeal, bladder, and rectal thermometer. 
Perioperative Predictors of Ultra-Fast-Track Failure and Prolonged Hospital Length of Stay in a Preliminary Protocol of Enhanced Recovery after off-Pump Coronary Artery Bypass Grafting

\section{Other Protocols}

The blood transfusion trigger is hemoglobin $(\mathrm{Hb})$ $\leq 8 / \mathrm{dL}$. Transfusion management is determined by hematocrit (Hct), ventricular function, oxygen saturation of central vein blood $\left(\mathrm{ScvO}_{2}\right)$, serum lactate, and co-morbidities, and the goal is to keep a Hct above $20-25 \%$ in patients with normal hemodynamic parameters $\left(\mathrm{ScvO}_{2}>70 \mathrm{mmHg}\right.$, and serum lactate $<2$ $\mathrm{mEq} / \mathrm{Lt}$ ), butabove $30 \%$ in patients with compromised hemodynamics, poor ventricular function ( $\mathrm{EF} \leq 30 \%)$, a new regional wall motion abnormality (RWMA), $\mathrm{ScvO}_{2}<70 \mathrm{mmHg}$, and serum lactate $>3 \mathrm{mEq} / \mathrm{Lt}$. Hourly urine output is registered through a Foley catheter. Anticoagulation therapy is performed at a heparin dose of $1.2 \mathrm{mg} / \mathrm{kg}$ IV to achieve an activated clotting time target of $300 \pm 20$ seconds. A tranexamic acid bolus $10 \mathrm{mg} / \mathrm{kg}$ IV is followed by $1 \mathrm{mg} / \mathrm{kg} / \mathrm{h} \mathrm{IV}$, and finally, heparin is reversed with protamine sulfate $\mathrm{IV}$, which is calculated at a dose of $1 \mathrm{mg}$ per every 100 units of heparin.

\section{Surgical Technique}

The myocardial revascularization is performed on the beating heart by the LAT using a tissue stabilization system. The patient is positioned with the left side elevated at approximately $45^{\circ}$, rotated decubitus toward the right with the help of a rolled towel. External pads for emergency defibrillation are placed on the right supero-anterior and left infero-posterior sides of the chest wall, and the incision is carried out on the fourth or fifth left intercostal space. The left internal mammary artery (LIMA) is harvested under direct visualization in a skeletonized fashion using a Finochietto retractor. If needed, the right internal mammary artery (RIMA) is harvested after the pericardial fat and thymus are carefully removed. When required, proximal graft anastomoses are performed using a side-bite partial clamp in the conventional fashion. The distal anastomoses are made on the beating heart using a pressure stabilizer and intracoronary shunt whenever possible. For the grafting of the circumflex and right coronary territory, the heart is lifted out of the pericardium using an apical suction device. ${ }^{10}$ Maintaining myocardial perfusion during the anastomosis is crucial since ventricular tachycardia or hemodynamic instability caused by myocardial ischemia could lead to an emergency conversion to sternotomy. ${ }^{8-12,16}$
Before leaving the Op.Room, fentanyl 0.5-1.0 $\mu \mathrm{g} / \mathrm{kg}$ is administered if necessary.

\section{Criteria for Extubation in the Op.Room}

Five respiratory criteria must be met no longer than 40 minutes for a patient to be eligible for extubation in the Op.Room. :

\section{A) Respiratory Criteria}

1. $\mathrm{Sp}_{02}>92 \%$ or $\mathrm{Pa}_{02}>60 \mathrm{mmHg}$;

2. spontaneous $R R>8$ bpm;

3. $\mathrm{ET}_{\mathrm{CO} 2}<50 \mathrm{mmHg}$ or $\mathrm{Pa}_{\mathrm{CO} 2}<55-60 \mathrm{mmHg}$;

4. Rapid Shallow Breathing Index < $105(R S B I=\mathrm{RR} / \mathrm{Vt})$;

5. No potential for difficult extubation (e.g., airway edema, difficult intubation)

Other manadatory requirements to meet for trying extubation in the Op.Room

B) Muscle relaxation status: TOF $>0.9$. (spontaneously, or sugammadex $2 \mathrm{mg} / \mathrm{kg}$ )

C) Neurologically intact (follows verbal commands, intact cough/gag reflex),

D) Acid-base status ( $\mathrm{pH}>7.35)$, normal electrolytes.

D)Hemodynamic status (IBP and PR $\pm 15-20 \%$ of baseline, non-threatening arrhythmias, without evident bleeding $(<50 \mathrm{ml} / \mathrm{h})$, and urinary output $>0.5 \mathrm{ml} / \mathrm{Kg} / \mathrm{h}$. Use of inotrope/vasopressor therapy is not an absolute contraindication. However, extubation is avoided if the vasoactive-inotropic score(VIS) is $\geq 10.16$

E) Normothermia (body temperature $36-37^{\circ} \mathrm{C}$ ) during the entire surgical procedure.

F) Pain management (visual analog scale $\leq 4 / 10$ ): Intercostals nerve block performed by surgeon with $20 \mathrm{~mL}$ bupivacaine $0.5 \%$ and $4 \mathrm{mg}$ of dexametasone at the level of the surgical approach, one level above and below the intercostal space used for the surgery. A multi-perforated epidural catheter through the incision and positioned in the involved intercostal space to administer a programmed continuous infusion of bupivacaine $0.5 \%$ at a rate of $10 \mathrm{ml} / \mathrm{hr}$ for the first $48 \mathrm{hrs}$. Rate its modified according a numeric pain rating scale .NSAIDS: ketoprofen $100 \mathrm{mg}$ IV and paracetamol 1gr IV are also used. Muscle relaxants are reversed with sugammadex at $2 \mathrm{mg} / \mathrm{kg}$ IV. Desflurane 
Perioperative Predictors of Ultra-Fast-Track Failure and Prolonged Hospital Length of Stay in a Preliminary Protocol of Enhanced Recovery after off-Pump Coronary Artery Bypass Grafting

and a remifentanil infusion are discontinued, and the patient is ventilated with $100 \%$ oxygen. Before extubation, the patient is then transported to the ICU in a $35^{\circ}$ sitting position, with a $50 \% \mathrm{O}_{2}$ face mask.

\section{Postoperative Care}

The patient is delivered to the ICU conscious, capable of responding to simple orders, and possibly with mild pain $(\mathrm{VAS}<4 / 10)$. For rescue pain relief, morphine is administered by patient-controlled analgesia consisting of a $1 \mathrm{mg}$ bolus of morphine with a15-minute lockout and maximal dose of $3 \mathrm{mg} / \mathrm{h}$. All infusions are checked. If the vital parameters are acceptable, cardiovascular drugs are gradually reduced. If the left lung is well re-expanded and tubes have stopped draining or $<200 \mathrm{ml} / 24 \mathrm{hrs}$ (Appendix 1), they are removed 12-24 hours postoperatively. If the routine blood tests and chest $\mathrm{x}$-ray are normal, a decision is made to discharge from the ICU. After ICU discharge, pain control is achieved with around-the-clock oral ibuprofen $400 \mathrm{mg}$ each 8 hours and paracetamol $1 \mathrm{~g}$ each 6 hours, along with omeprazole 40mg/ day. The patient is then transferred to continuous cardiac telemetry monitoring and the appropriate cardiovascular medications are administered. The intercostal catheter is discontinued 4-6 hrs before hospital discharge, and tramadol drops $(0.5-1 \mathrm{mg} / \mathrm{kg}$ each 6-8 hours) are used as rescue pain relief.

Once discharged, the patient is given the telephone number of a surgical team member (surgeon coordinator) in case there are any symptoms or complications. During ERAS interview before the surgery, the patient and family would have been taught how to perform respiratory exercises and manage wounds, and the patient, encouraged to start walking as soon as possible (Appendix 1). A team of nurses performs daily at-home wound care for a week. The patient is then followed with regular outpatient appointments with either the surgeon or cardiologist at $1,2,4,6$, and 8 weeks postoperatively.

\section{Statistical Analysis}

Analyses were performed using SPSS version 16.0 for Windows (SPSS,Chicago,IL,USA).

Descriptivestatistic: Demographicand clinicalvariables were described by means, standard deviations, median, mode, ranges (min-max) for continuous variables, and absolute and relative frequency (\%) for categorical variables. Many of the variables were presented as dichothomous. Age was redefined as a dichotomous variable using the cut-off of 80 years, to calculate odds ratios for age.(Appendix 2).

Inferential statistic: to look for predictors (risk factors) for UTF-failure and prolonged Hosp-LOS:

$1^{\text {st }}$ Step) Estimation of Crude (no adjusted) Odds Ratio(OR) with 95\% confidential interval (CI). Univariate logistic regression analysis was used to evaluate association between each independent variable with extubation outcome (UFT-failure) and prolonged Hosp-LOS, defined as $>4$ days.

$2^{\text {nd }}$ Ste $p$ ) Estimation of Adjusted OR (AOR): Multivariate binary logistic regression model was used including all significant variables $(p<0.05$ or with a statistic tendency, $\mathrm{p}<0.1$ ) obtained in $1^{\text {st }}$ step.

$3^{\text {rd }}$ Step) Backward elimination method to identify the independent variables with enough explanatory ability. The alpha level was set at $\mathrm{p}<0.05(5 \%)$ and power at $80 \%$.

\section{RESULTS}

A total of 673 consecutive patients who were 518 male (77\%), 155 female(23\%) and a mean age of $61.5 \pm$ 9.5 years old (y.o), median 61 y.o (range, 36-90 years) were included in the study. All had undergone OPCAB by the same surgical, anesthetic, and critical care team. The mean Euro SCORE was $5.2 \pm 4.1$ (range, 0 -17). The NYHA functional classification was Class I, 72 patients (10.7\%); Class II, 385 (57.2\%); Class III, 150 (22.3\%) and Class IV, 66 (9.8\%). The mean ejection fraction (EF) was $47 \pm 10 \%, 335$ patients (49.8\%) of patients had $\mathrm{EF}>50 \%$ and 41 patients (6.1\%) had $\mathrm{EF}<30 \%$. Coronary artery disease affected three vessels in 580 patients (86.2\%); within this group, 57 (8.4\%) required four or more grafts. A majority $(546,81.1 \%)$ were diagnosed with hypertension and $412(61.2 \%)$ were taking two or more antihypertensive medications. A total of $456(67.7 \%)$ had hyperlpidemia and close to half $(41 \%)$ had diabetes mellitus type 2.None patient had DM tipo I. 168 patients had obesity with BMI $30-37 \mathrm{Kg} / \mathrm{mt}^{2}$, the majority were Class I(BMI 30-34 $\mathrm{Kg} / \mathrm{mt}^{2}$ ) following the World Health Organization classification. There were 215 (31.9\%) with chronic obstructive pulmonary disease. Twice as many (446, $66.3 \%$ ) smoked 10 or more cigarettes per day. Nearly half had experienced a previous myocardial infarction. (Table 1) 
Perioperative Predictors of Ultra-Fast-Track Failure and Prolonged Hospital Length of Stay in a Preliminary Protocol of Enhanced Recovery after off-Pump Coronary Artery Bypass Grafting

Table1. Short-Time Outcome. Descriptive data. Demographic, clinical and surgical variables. $N$ (\%) / mean \pm standard desviation (sd).

\begin{tabular}{|c|c|}
\hline & Total \\
\hline & $\mathrm{N}(\%) /$ mean \pm sd \\
\hline Patients & $673(100)$ \\
\hline AGE (y.o) & $61.5 \pm 9.5$ \\
\hline \multirow[t]{2}{*}{ GROUP AGE } & $641(95.2)$ \\
\hline & $32(4.8)$ \\
\hline GENDER & $518(77)$ \\
\hline Female & $155(23)$ \\
\hline SMOKER & $227(33.7)$ \\
\hline Yes & $446(66.3)$ \\
\hline \multirow[t]{4}{*}{ NYHA } & $72(10.7)$ \\
\hline & $385(52.7)$ \\
\hline & $150(22.3)$ \\
\hline & $66(9.8)$ \\
\hline \multirow[t]{3}{*}{ CAD } & $40(5.9)$ \\
\hline & $53(7.9)$ \\
\hline & $580(86.2)$ \\
\hline \multirow{2}{*}{ LEFT MAIN STEM } & $547(81.3)$ \\
\hline & $126(18.7)$ \\
\hline \multirow[t]{4}{*}{ A. P(CCS) } & $355(52.7)$ \\
\hline & $144(21.4)$ \\
\hline & $127(18.9)$ \\
\hline & $47(7.0)$ \\
\hline \multirow[t]{2}{*}{ HTA } & $127(18.9)$ \\
\hline & $546(81.1)$ \\
\hline LV FUNCTION (EF) & $47.3 \pm 10.2$ \\
\hline \multirow[t]{3}{*}{ GROUP LV (EF) } & $335(49.8)$ \\
\hline & $297(44.1)$ \\
\hline & $41(6.1)$ \\
\hline \multirow[t]{2}{*}{ MYOCARDIAL INFARCTION } & $342(50.8)$ \\
\hline & $\begin{array}{l}331(49.2) \\
148(21.9 \%)\end{array}$ \\
\hline \multirow[t]{2}{*}{ PRE-STROKE } & $617(91.7)$ \\
\hline & $56(8.3)$ \\
\hline \multirow[t]{2}{*}{ REDO } & $645(95.8)$ \\
\hline & $28(4.2)$ \\
\hline \multirow[t]{2}{*}{ PAD } & $448(66.6)$ \\
\hline & $225(33.4)$ \\
\hline \multirow[t]{2}{*}{ DM II } & $397(59.0)$ \\
\hline & $276(41.0)$ \\
\hline \multirow[t]{2}{*}{ HYPERLIPIDEMA } & $217(32.2)$ \\
\hline & $456(67.8)$ \\
\hline \multirow[t]{2}{*}{ OBESITY (BMI>30 WHO) } & $505(75.0)$ \\
\hline & $168(25.0)$ \\
\hline \multirow[t]{2}{*}{ RENAL.F (Creatinine $>2.0 \mathrm{mg} / \mathrm{dl}$ ) } & $568(94.4)$ \\
\hline & $105(15.6)$ \\
\hline COPD (GOLD) & $458(68.1)$ \\
\hline
\end{tabular}


Perioperative Predictors of Ultra-Fast-Track Failure and Prolonged Hospital Length of Stay in a Preliminary Protocol of Enhanced Recovery after off-Pump Coronary Artery Bypass Grafting

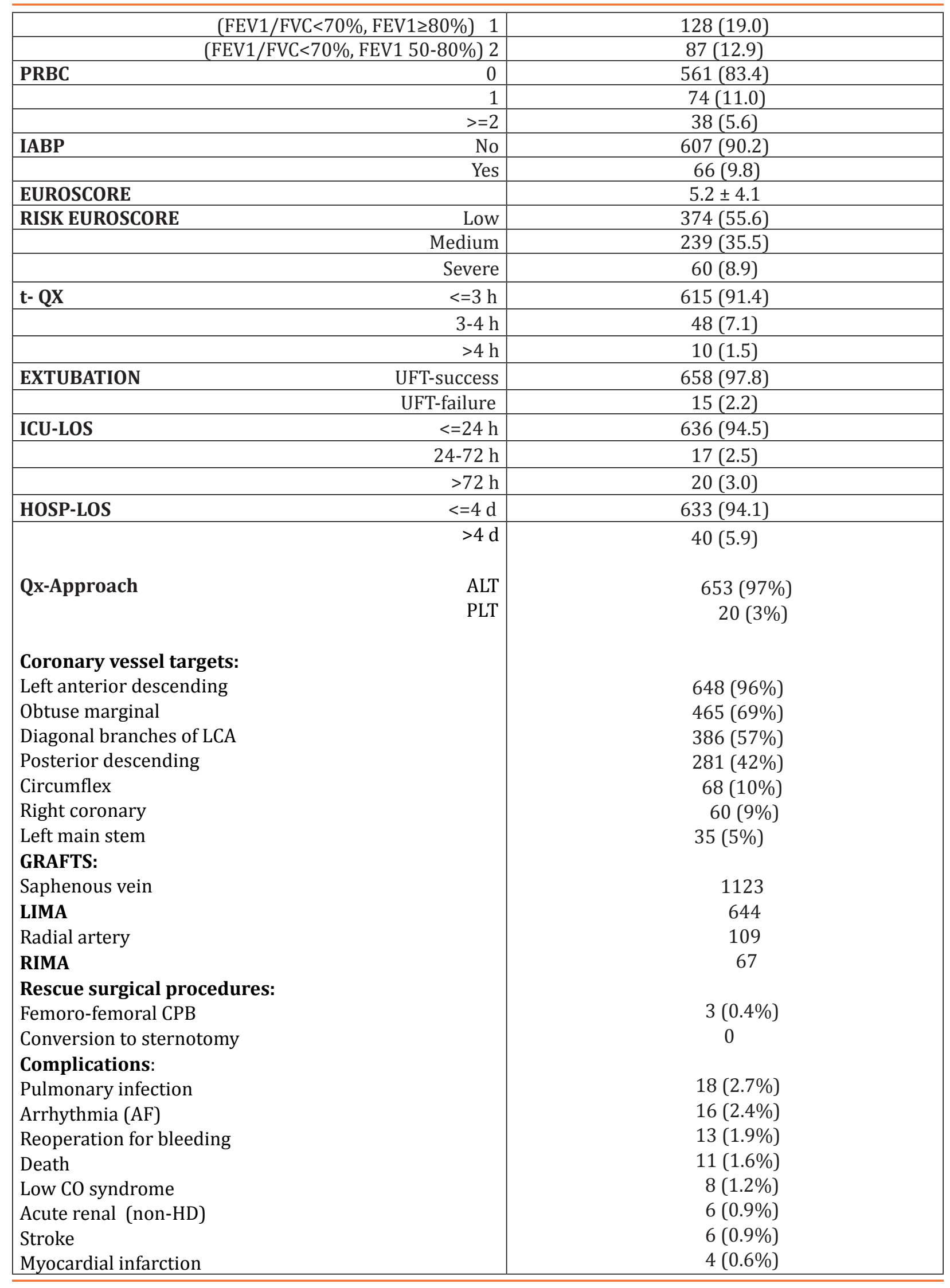

Archives of Anesthesiology V1 . I2 . 2018 
Perioperative Predictors of Ultra-Fast-Track Failure and Prolonged Hospital Length of Stay in a Preliminary Protocol of Enhanced Recovery after off-Pump Coronary Artery Bypass Grafting

UFT-success: extubation in Op.Room. UFTfailure=Extubation in ICU. NYHA: New York Heart Association risk scale. HTA: hypertension, CAD: coronary artery disease. A.P(CCS): angina pectoris Canadian Cardiovascular Society grades. LV: Left Ventricular, EF: Ejection Fraction, PCI: previous percutaneous coronary intervention, PAD: peripheral artery disease, DM II: diabetes mellitus type II, RENAL F.: Renal failure REDO: reintervention,CABG: Coronary Artery Bypass Grafting, COPD :Chronic Obstructive Pulmonary Disease, GOLD: The Global Iniciative for Chronic Obstructive Lung Disease staging system. IABP:preoperative Intra-Aortic Balloon Pump., PRBC: Packet red blood cell units transfused. t-Qx: surgery duration, ICU-LOS: Intensive critical unit length of stay, HOSP-LOS: hospitalization length of stay. QxApproach: Surgical approach. ALT. antero-lateral thoracotomy, PLT: posterolateral thoracotomy. LIMA : Left Internal Mammary Artery, RIMA: Right Internal Mammary Artery CBP: cardio pulmonary bypass Op.Room: Operative Room, ICU: Intensive Critical Unit., AF: Atrial Fibrillation, HD: Haemodialysis

In all 1,943 bypasses were performed with an average of $3 \pm 1$ bypass grafts per patient. The approach was by LAT in 653 patients (97\%) and posterolateral thoracotomy in 20 (3\%). 91.4\% of patient had a surgery time $(\mathrm{t}-\mathrm{Qx}) \leq 3 \mathrm{hrs}$ and 58 patients $(8.6 \%)>3$ hrs. Sixty-six patients $(9.8 \%)$ received elective intraaortic balloon pump (IABP) because of having met two or more of following conditions: left main stem disease, unstable angina pectoris, myocardial infarction $\leq 30$ days, redo, and $\mathrm{EF} \leq 30 \%$. No cases converted to sternotomy. Three patients required hemodynamic support by femoro-femoro cardiopulmonary bypass (CBP) although the beating heart was kept during coronary arteries bypasses placement (Table 1)

\section{Postoperative Outcomes}

Nearly all patients $(658,97.8 \%)$ were extubated in the Op.Room whereas only 15 (2.2\%) were extubated later in the ICU (UFT-failure), for the most part because of bleeding. Three patients $(0.5 \%)$ were reintubated within the first 72 hours in the ICU. The mean ICULOS was $29 \pm 4.2$ hours, mode 20 hrs (range, 8-480 hours), and nearly all patients $(636,94.5 \%)$ were in the ICU for less than 24 hours. The ICU readmission rate was $2.1 \%$ (14 patients). Three patients were readmitted twice, and one patient three times; all four died. The most common reasons for ICU readmission were pneumonia (9 patients), sepsis (9), acute respiratory distress syndrome (6), and multiorgan failure syndrome (5). The mean of Hosp-LOS after discharge from the ICU was $50.5 \pm 9.9$ hours, mode 48 hrs (range, 22-528 hours).

633 patient had Hosp-LOS $\leq 4$ days (94.1\%) and 5.9\% with Hosp-LOS $>4$ days. Overall, the more common complications were pulmonary infection $(18,2.7 \%)$, arrhythmia (16, 2.4\%), and reoperation for bleeding $(13,1.9 \%)$ (Table1). Mean volume of blood lost was $541.5 \pm 61.3 \mathrm{~mL}$ (range, 63-3500 mL); 112 (16.6\%) patients received a mean of $1.0 \pm 2.5$ PRBC units (range,0-8 Units p.p); 74 patients were transfused with 1 PRBC units and 38 patients (5.6\%) received $\geq 2$ PRBC. Eleven patients (1.6\%) died within 30 days of the procedure. Those who died were diagnosed with multiple complications, the most common of which were multiorgan failure syndrome (9), acute respiratory distress syndrome (8), pneumonia (6), and sepsis (6).

Univariate analysis revealed as independent risk factors for UFT-failure: age, female sex, EuroSCORE, Severity of Angina Pectoris (IV-grade CCS), EF<30\%, Redo, COPD, PRBC transfusion, use of elective IABP and duration of surgery(t-Qx). Redo was strongly significant (OR=36.5, $\mathrm{p}<0.001)$. (Table 2).

Table 2. Extubation Outcome. Descriptive data: $N(\%)$ or media \pm standard desviation(sd) . Univariate binary logistic regression for each variable: No adjusted Odds Ratio (OR), 95\%(CI) and p-value.

\begin{tabular}{|l|c|c|c|c|c|}
\hline & \multicolumn{2}{|c|}{ EXTUBATION } & \multicolumn{2}{|c|}{} \\
\cline { 1 - 4 } & UFT-success & UFT-failure & \multicolumn{2}{|c}{ OR (95\%CI) } & p-value \\
\hline Patients & \multicolumn{2}{|c|}{$\mathrm{N}(\%) /$ mean \pm sd } & $15(2.2)$ & & \\
\hline AGE (y) & $658(97.8)$ & $61.3 \pm 9.4$ & $73.1 \pm 9.8$ & $1.13(1.07-1.19)$ & $<\mathbf{0 . 0 0 1 * * *}$ \\
\hline
\end{tabular}


Perioperative Predictors of Ultra-Fast-Track Failure and Prolonged Hospital Length of Stay in a Preliminary Protocol of Enhanced Recovery after off-Pump Coronary Artery Bypass Grafting

\begin{tabular}{|c|c|c|c|c|}
\hline GROUP AGE & $630(98.3)$ & $11(1.7)$ & 1 & \\
\hline$>80 \mathrm{y}$ & $28(87.5)$ & $4(12.5)$ & $8.18(2.45-27.3)$ & $0.001 * *$ \\
\hline \multirow[t]{2}{*}{ GENDER } & $515(99.4)$ & $3(0.6)$ & 1 & \\
\hline & $143(92.3)$ & $12(7.7)$ & $14.4(4.01-51.7)$ & $<0.001^{* * *}$ \\
\hline \multirow[t]{2}{*}{ SMOKER } & $219(96.5)$ & $8(3.5)$ & 1 & \\
\hline & $439(98.4)$ & $7(1.6)$ & $0.44(0.16-1.22)$ & 0.114 \\
\hline \multirow[t]{4}{*}{ NYHA } & $68(94.4)$ & $4(5.6)$ & 1 & 0.082 \\
\hline & $381(99.0)$ & $4(1.0)$ & $0.18(0.04-0.73)$ & 0.017* \\
\hline & $146(97.3)$ & $4(2.7)$ & $0.47(0.11-1.92)$ & 0.290 \\
\hline & $63(95.5)$ & $3(4.5)$ & $0.81(0.17-3.76)$ & 0.787 \\
\hline \multirow[t]{3}{*}{ CAD } & $40(100)$ & $0(0)$ & \multirow{2}{*}{ 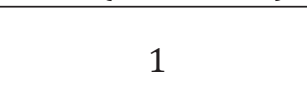 } & \\
\hline & $51(96.2)$ & $2(3.8)$ & & \\
\hline & $567(97.8)$ & $13(2.2)$ & $1.04(0.23-4.70)$ & 0.956 \\
\hline \multirow[t]{2}{*}{ LEFT MAIN STEM } & $536(98.0)$ & $11(2.0)$ & 1 & \\
\hline & $122(96.8)$ & $4(3.2)$ & $1.59(0.50-5.10)$ & 0.429 \\
\hline \multirow[t]{4}{*}{$\mathrm{AP}(\mathrm{CCS})$} & $353(99.4)$ & $2(0.6)$ & 1 & 0.016* \\
\hline & $140(97.2)$ & $4(2.8)$ & $5.04(0.91-27.8)$ & 0.063 \\
\hline & $122(96.1)$ & $5(3.9)$ & $7.23(1.39-37.7)$ & 0.019* \\
\hline & $43(91.5)$ & $4(8.5)$ & $16.4(2.92-92.3)$ & 0.001** \\
\hline \multirow[t]{2}{*}{ HTA } & $119(93.7)$ & $8(6.3)$ & 1 & \\
\hline & $539(98.7)$ & $7(1.3)$ & $0.19(0.07-0.54)$ & $0.002^{* *}$ \\
\hline LV FUNCTION (EF) & $47.4 \pm 10.2$ & $41.9 \pm 12.1$ & $0.95(0.91-0.99)$ & $0.046^{*}$ \\
\hline GROUP LV FUNCTION (EF) & $331(98.8)$ & $4(1.2)$ & 1 & $0.009 * *$ \\
\hline $30-50 \%$ & $290(97.6)$ & $7(2.4)$ & $1.99(0.58-6.89)$ & 0.274 \\
\hline$<30 \%$ & $37(90.2)$ & $4(9.8)$ & $8.94(2.14-37.2)$ & $0.003 * *$ \\
\hline \multirow[t]{2}{*}{ MYOCARDIAL INFARCTION } & $336(98.2)$ & $6(1.8)$ & 1 & \\
\hline & $322(97.3)$ & $9(2.7)$ & $1.56(0.55-4.45)$ & 0.400 \\
\hline \multirow[t]{2}{*}{ PRE-STROKE } & $603(97.7)$ & $14(2.3)$ & 1 & \\
\hline & $55(98.2)$ & $1(1.8)$ & $0.78(0.10-6.07)$ & 0.815 \\
\hline \multirow[t]{2}{*}{ REDO } & $638(98.9)$ & $7(1.1)$ & 1 & \\
\hline & $20(71.4)$ & $8(28.6)$ & $36.5(12.0-110.3)$ & $<0.001^{* * *}$ \\
\hline \multirow[t]{2}{*}{ PAD } & $440(98.2)$ & $8(1.8)$ & 1 & \\
\hline & $218(96.9)$ & $7(3.1)$ & $1.77(0.63-4.93)$ & 0.278 \\
\hline \multirow[t]{2}{*}{ DM II } & $387(97.5)$ & $10(2.5)$ & 1 & \\
\hline & $271(98.2)$ & $5(1.8)$ & $0.71(0.24-2.11)$ & 0.543 \\
\hline \multirow[t]{2}{*}{ HYPERLIPIDEMA } & $212(97.7)$ & $5(2.3)$ & 1 & \\
\hline & $446(97.8)$ & $10(2.2)$ & $0.95(0.32-2.82)$ & 0.927 \\
\hline \multirow{2}{*}{$\begin{array}{rr}\text { OBESITY } & \text { No } \\
& \text { Yes } \\
\end{array}$} & $494(97.8)$ & $11(2.2)$ & 1 & \\
\hline & $164(97.6)$ & $4(2.4)$ & $1.09(0.34-3.49)$ & 0.877 \\
\hline $\begin{array}{r}\text { RENAL failure (Creatinine }>2.0 \mathrm{mg} / \mathrm{d} \text { ) } \\
\text { No }\end{array}$ & $556(97.9)$ & $12(2.1)$ & 1 & \\
\hline Yes & $102(97.1)$ & $3(2.9)$ & $1.36(0.38-4.91)$ & 0.636 \\
\hline
\end{tabular}


Perioperative Predictors of Ultra-Fast-Track Failure and Prolonged Hospital Length of Stay in a Preliminary Protocol of Enhanced Recovery after off-Pump Coronary Artery Bypass Grafting

\begin{tabular}{|c|c|c|c|c|c|}
\hline COPD & No & $451(98.5)$ & $7(1.5)$ & 1 & 0.072 \\
\hline & 1 & $125(97.7)$ & $3(2.3)$ & $1.55(0.39-6.07)$ & 0.532 \\
\hline & 2 & $82(94.3)$ & $5(5.7)$ & $3.93(1.22-12.7)$ & $0.022^{*}$ \\
\hline \multirow[t]{3}{*}{ PRBC } & 0 & $560(99.8)$ & $1(0.2)$ & 1 & $<0.001^{* * *}$ \\
\hline & 1 & $72(97.3)$ & $2(2.7)$ & $15.6(139-173.0)$ & $0.026^{*}$ \\
\hline & $>=2$ & $26(68.4)$ & $12(31.6)$ & $258.5(32.3-2063)$ & $<0.001^{* * *}$ \\
\hline \multirow[t]{2}{*}{ IABP } & No & $597(98.4)$ & $10(1.6)$ & 1 & \\
\hline & Yes & $61(92.4)$ & $5(7.6)$ & $4.89(1.62-14.8)$ & $0.005^{* *}$ \\
\hline EUROSCORE & & $5.1 \pm 3.9$ & $11.8 \pm 5.4$ & $1.34(1.20-1.49)$ & $<0.001 * * *$ \\
\hline \multirow[t]{3}{*}{ RISK EUROSCORE } & Low & 373 (99.7) & $1(0.3)$ & 1 & $<0.001 * * *$ \\
\hline & Medium & $233(97.5)$ & $6(2.5)$ & $9.61(1.15-80.2)$ & $0.037^{*}$ \\
\hline & Severe & $52(86.7)$ & $8(13.3)$ & $57.4(7.03-468.1)$ & $<0.001 * * *$ \\
\hline \multirow[t]{3}{*}{$t-Q x$} & $<=3 \mathrm{~h}$ & $614(94.8)$ & $1(0.2)$ & 1 & $<0.001 * * *$ \\
\hline & $3-4 \mathrm{~h}$ & $38(79.2)$ & $10(20.8)$ & $161.6(20.1-1295.4)$ & $<0.001^{* * *}$ \\
\hline & $>4 \mathrm{~h}$ & $6(60.0)$ & $4(40.0)$ & $409.3(39.7-4224.7)$ & $<0.001^{* * *}$ \\
\hline
\end{tabular}

${ }^{*} \mathrm{p}<0.05 ; \quad{ }^{* *} \mathrm{p}<0.01 ; \quad * * * \mathrm{p}<0.001$

UFT-success: extubation in Op.Room. UFT-failure= Extubation in ICU. NYHA: New York Heart Association risk scale. HTA: hypertension, CAD: coronary artery disease. A.P(CCS): angina pectoris Canadian Cardiovascular Society grades. LV: Left Ventricular, EF: Ejection Fraction, PCI: previous percutaneous coronary intervention, PAD: peripheral artery disease ,DM II: diabetes mellitus type II, REDO: reintervention, CABG: CoronaryArtery Bypass Grafting, COPD:Chronic Obstructive Pulmonary Disease IABP: preoperative Intra-Aortic Balloon Pump., PRBC: Packet red blood

cell units transfused. $\mathbf{t}-\mathbf{Q x}$ : surgery duration, ICULOS: Intensive critical unit length of stay, HOSP-LOS: hospitalization length of stay Op.Room: Operative Room, ICU: Intensive Critical Unit

After performing multivariate logistic regression analysisandbackward eliminationmethod, thestronger predictor factors for UFT-failure were: transfusion of $\geq 2$ PRCB revealed Adjusted OR (AOR=6.02) $(95 \% \mathrm{CI})$ $(\mathrm{p}<0.05), \mathrm{t}-\mathrm{Q} x \mathrm{3}-4 \mathrm{hrs},(\mathrm{AOR}=77.3)(95 \% \mathrm{CI})(\mathrm{p}<0.001)$ and $\mathrm{t}-\mathrm{Qx}>4 \mathrm{hrs},(\mathrm{AOR}=157.5)(95 \% \mathrm{CI})(\mathrm{p}<0.001) . \mathrm{EF}$ $<30 \%$ had (OR=8.41) $(95 \% \mathrm{CI})(\mathrm{p}=0.059)$. (Table 3$)$.

Table 3. Independent variables associated with UFT-failure. Multiple binary logístic regression : Adjusted odds ratio (AOR) by backward elimination method.95\%(CI) and p-value.

\begin{tabular}{|c|c|c|}
\hline & \multicolumn{2}{|c|}{ UFT-failure } \\
\hline & AOR (95\%CI) & $\mathrm{p}$-value \\
\hline A. P(CCS) & 1 & $0.035 *$ \\
\hline Grade IV-A & $6.18(0.82-46.5)$ & 0.077 \\
\hline Grade IV-B & $12.0(1.66-87.3)$ & 0.014* \\
\hline Grade IV-C & $0.58(0.05-6.44)$ & 0.054* \\
\hline GROUP LV FUNCTION (EF) & 1 & \\
\hline$<30 \%$ & $8.41(0.92-76.7)$ & 0.059 \\
\hline PRBC & 1 & \\
\hline$>=2$ & $6.02(1.02-35.4)$ & $0.047 *$ \\
\hline$<=3 \mathrm{~h}$ & 1 & $0.002^{* *}$ \\
\hline $3-4 \mathrm{~h}$ & $77.3(6.04-991.4)$ & $0.001^{* *}$ \\
\hline$>4 \mathrm{~h}$ & $157.5(8.73-2843.2)$ & $0.001 * *$ \\
\hline
\end{tabular}

${ }^{*} \mathrm{p}<0.05 ; \quad{ }^{* *} \mathrm{p}<0.01 ; \quad * * * \mathrm{p}<0.001$ 
Perioperative Predictors of Ultra-Fast-Track Failure and Prolonged Hospital Length of Stay in a Preliminary Protocol of Enhanced Recovery after off-Pump Coronary Artery Bypass Grafting

UFT-failure: extubation in UCI. A.P(CCS): angina pectoris Canadian Cardiovascular Society grades. LV: Left Ventricular, EF: Ejection Fraction PRBC: Packet red blood cell units transfused. t-Qx: surgery duration

Univariate analysis revealed as significant predictors or independent risk factors for Hosp-LOS $>4$ days
: Age $>80$ yo ,female sex, NYHA $>$ III, EurosCORE, severity of Angina pectoris CCS, $\mathrm{EF}<30 \%$, Redo, Renal failure, IABP, PRCB transfusion, UFT-failure failure, $\mathrm{t}-\mathrm{Qx}$ and ICU-LOS $(\mathrm{p}<0.001)$. Transfusion of 1 PRCB increased 48.8 times the risk of Hops-LOS $>4$ days, and transfusion of $\geq 2$ PRCB showed (OR=686) $(95 \% \mathrm{CI})$. (Table 4).

Table 4. Independent variables associated with Hospital length of Stay (HOSP-LOS). Descriptive data: N (\%) or media \pm standard desviation (sd). Univariate binary logistic regression for each variable: No adjusted Odds Ratio (OR), 95\%(CI) and p-value.

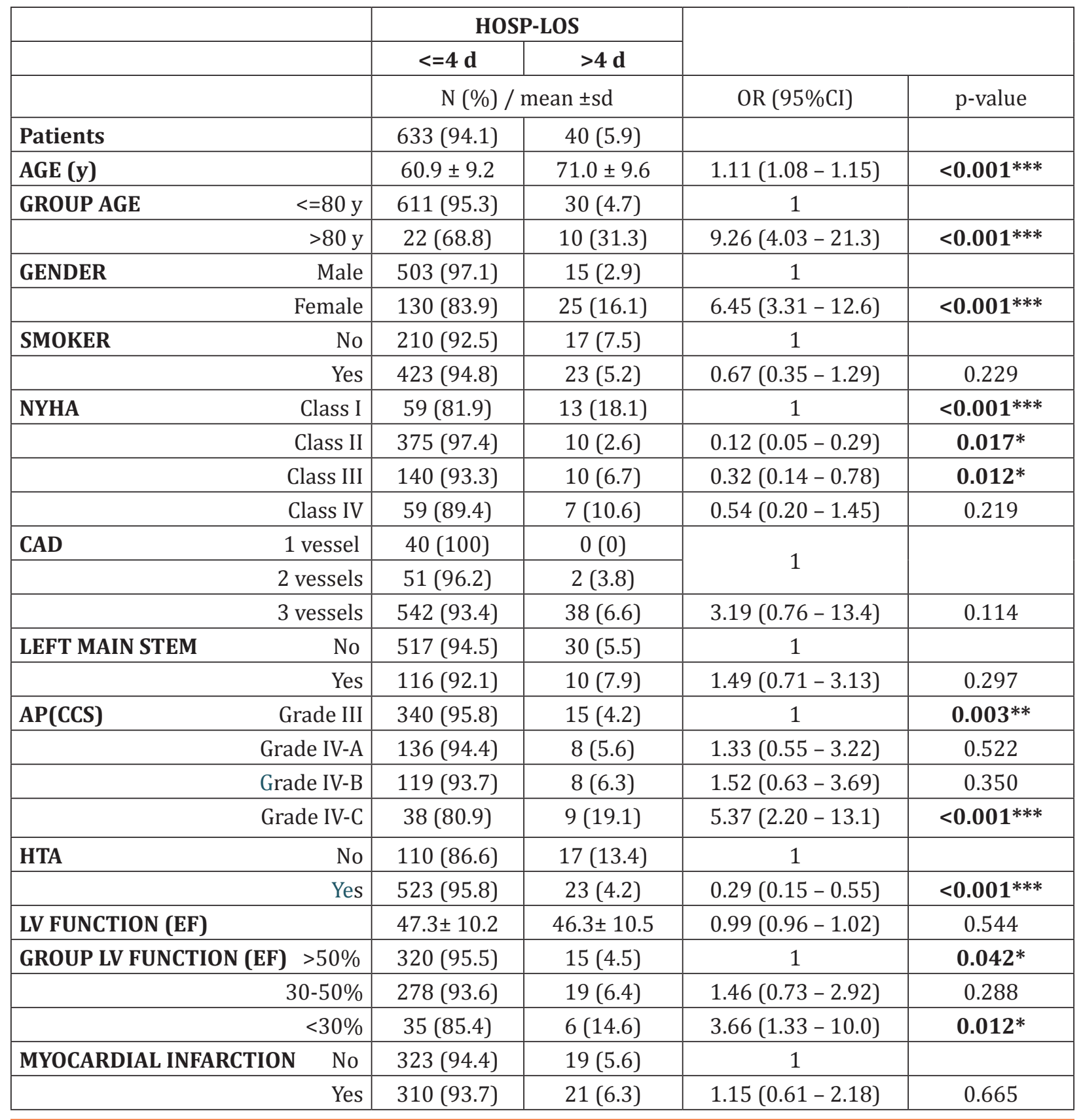


Perioperative Predictors of Ultra-Fast-Track Failure and Prolonged Hospital Length of Stay in a Preliminary Protocol of Enhanced Recovery after off-Pump Coronary Artery Bypass Grafting

\begin{tabular}{|c|c|c|c|c|c|}
\hline PRE-STROKE & No & $581(94.2)$ & $36(5.8)$ & 1 & \\
\hline & Yes & $52(92.9)$ & $4(7.1)$ & $1.24(0.43-3.62)$ & 0.692 \\
\hline \multirow[t]{2}{*}{ REDO } & No & $629(97.5)$ & $16(2.5)$ & 1 & \\
\hline & Yes & $4(14.3)$ & $24(85.7)$ & $235.9(73.2-759.2)$ & $<0.001^{* * *}$ \\
\hline \multirow[t]{2}{*}{ PAD } & No & $425(94.9)$ & $23(5.1)$ & 1 & \\
\hline & Yes & $208(92.4)$ & $17(7.6)$ & $1.51(0.79-2.89)$ & 0.213 \\
\hline \multirow[t]{2}{*}{ DM II } & No & $373(94.0)$ & $24(6.0)$ & 1 & \\
\hline & Yes & $260(94.2)$ & $16(5.8)$ & $0.96(0.50-1.84)$ & 0.893 \\
\hline \multirow[t]{2}{*}{ HYPERLIPIDEMA } & No & $202(93.1)$ & $15(6.9)$ & 1 & \\
\hline & Yes & $431(94.5)$ & $25(5.5)$ & $0.78(0.40-1.51)$ & 0.464 \\
\hline \multirow[t]{2}{*}{ OBESITY } & No & $478(94.7)$ & $27(5.3)$ & 1 & \\
\hline & Yes & $155(92.3)$ & $13(7.7)$ & $1.49(0.75-2.95)$ & 0.259 \\
\hline \multirow[t]{2}{*}{ RENAL FAILURE } & No & $543(95.6)$ & $25(4.4)$ & 1 & \\
\hline & Yes & $90(85.7)$ & $15(14.3)$ & $3.62(1.84-7.13)$ & $<0.001 * * *$ \\
\hline \multirow[t]{3}{*}{ COPD } & No & $436(95.2)$ & $22(4.8)$ & 1 & 0.194 \\
\hline & 1 & $117(91.4)$ & $11(8.6)$ & $1.86(0.88-3.95)$ & 0.105 \\
\hline & 2 & $80(92.0)$ & $7(8.0)$ & $1.73(0.72-4.19)$ & 0.222 \\
\hline \multirow[t]{3}{*}{ PRBC } & 0 & $559(99.6)$ & $2(0.4)$ & 1 & $<0.001^{* * *}$ \\
\hline & 1 & $63(85.1)$ & $11(14.9)$ & $48.8(10.6-225.1)$ & $<0.001^{* * *}$ \\
\hline & $>=2$ & $11(28.9)$ & $27(71.1)$ & $686.0(144.8-3249)$ & $<0.001^{* * *}$ \\
\hline \multirow[t]{2}{*}{ IABP } & No & $576(94.9)$ & $31(5.1)$ & 1 & \\
\hline & Yes & $57(86.4)$ & $9(13.6)$ & $2.93(1.33-6.47)$ & $0.008^{* *}$ \\
\hline EUROSCORE & & $4.9 \pm 3.9$ & $9.3 \pm 4.9$ & $1.24(1.15-1.32)$ & $<0.001^{* * *}$ \\
\hline \multirow[t]{3}{*}{ RISK EUROSCORE } & Low & $365(97.6)$ & $9(2.4)$ & 1 & $<0.001^{* * *}$ \\
\hline & Medium & $219(91.6)$ & $20(8.4)$ & $3.70(1.66-8.28)$ & $0.001^{* *}$ \\
\hline & Severe & $49(81.7)$ & $11(18.3)$ & $9.10(3.59-23.1)$ & $<0.001 * * *$ \\
\hline \multirow[t]{3}{*}{$\mathrm{t}-\mathrm{QX}$} & $<=3 \mathrm{~h}$ & $611(99.3)$ & $4(0.7)$ & 1 & $<0.001^{* * *}$ \\
\hline & $3-4 \mathrm{~h}$ & $20(41.7)$ & $28(58.3)$ & $213.9(68.5-667.6)$ & $<0.001 * * *$ \\
\hline & $>4 \mathrm{~h}$ & $2(20.0)$ & $8(80.0)$ & $611.0(97.5-3828.4)$ & $<0.001 * * *$ \\
\hline \multirow[t]{2}{*}{ EXTUBATION } & UFT-success & $628(95.4)$ & $30(4.6)$ & 1 & \\
\hline & UFT-failure & $5(33.3)$ & $10(66.7)$ & $41.9(13.5-130.2)$ & $<0.001^{* * *}$ \\
\hline \multirow[t]{3}{*}{ ICU-LOS } & $<=24 \mathrm{~h}$ & $628(98.7)$ & $8(1.3)$ & 1 & \\
\hline & $>24-72 \mathrm{~h}$ & $5(29.4)$ & $12(70.6)$ & \multirow{2}{*}{$502.4(155.5-1622)$} & \multirow{2}{*}{$<0.001^{* * *}$} \\
\hline & $>72 \mathrm{~h}$ & $0(0.0)$ & $20(100)$ & & \\
\hline
\end{tabular}

${ }^{*} \mathrm{p}<0.05 ; \quad * * \mathrm{p}<0.01 ; \quad * * * \mathrm{p}<0.001$

UFT-success: extubation in Op.Room. UFT-failure= Extubation in ICU. NYHA: New York Heart Association risk scale.HTA: hypertension, CAD: coronary artery disease. A.P(CCS): Angina Pectoris Canadian Cardiovascular Society grades. LV: Left Ventricular, EF: Ejection Fraction, PCI: previouspercutaneouscoronary intervention, PAD: peripheral artery disease, DM II: diabetes mellitus type II, REDO: reintervention, CABG:
Coronary Artery Bypass Grafting, COPD : Chronic Obstructive Pulmonary Disease IABP: preoperative Intra-Aortic Balloon Pump., PRBC: Packet red blood cell units transfused. t-Qx: surgery duration, ICULOS: Intensive critical unit length of stay, HOSP-LOS: hospitalization length of stay Op.Room: Operative Room, ICU: Intensive Critical Unit 
Perioperative Predictors of Ultra-Fast-Track Failure and Prolonged Hospital Length of Stay in a Preliminary Protocol of Enhanced Recovery after off-Pump Coronary Artery Bypass Grafting

Multivariate analysis and backward elimination method revealed as stronger predictor factors for Hosp-LOS>4 days the following variables:

LOS $>24$ hrs $(A O R=29.3)(95 \% \mathrm{CI})(\mathrm{p}<0.001)$ and Redo (AOR=7.68) $(95 \% \mathrm{CI})$ with an important statistic tendency ( $\mathrm{p}=0.059)$.. Preoperative use of IABP reduce risk of Hosp $>4$ days , (AOR=0.06, 0.01-0.51) $((\mathrm{p}=0.01)$. $\mathrm{t}-\mathrm{Qx}>3 \mathrm{hrs}(\mathrm{AOR}>74)(95 \% \mathrm{CI})(\mathrm{p}<0.001)$ and ICU- (Table 5)

Table 5. Independient risk factors associated with Hosp-LOS $>4$ days Multiple binary logístic regression : Adjusted odds ratio (AOR) by backward elimination method, 95\%(CI) and p-valor.

\begin{tabular}{|c|c|c|c|}
\hline & & \multicolumn{2}{|c|}{ HOSP-LOS $>4$ days } \\
\hline & & AOR $(95 \% \mathrm{CI})$ & $p$-value \\
\hline \multirow[t]{2}{*}{ REDO } & No & 1 & \\
\hline & Yes & $7.68(0.93-63.4)$ & 0.059 \\
\hline \multirow[t]{2}{*}{ IABP } & No & 1 & \\
\hline & Yes & $0.06(0.01-0.51)$ & 0.010* \\
\hline \multirow[t]{3}{*}{$t-Q X$} & $<=3 \mathrm{~h}$ & 1 & $<0.001^{* * *}$ \\
\hline & $3-4 \mathrm{~h}$ & $74.2(15.4-358.2)$ & $<0.001^{* * *}$ \\
\hline & $>4 \mathrm{~h}$ & $95.1(3.96-2285.9)$ & $0.005^{* *}$ \\
\hline \multirow[t]{2}{*}{ ICU-LOS } & $<=24 \mathrm{~h}$ & 1 & \\
\hline & $>24 \mathrm{~h}$ & $29.3(4.44-193.1)$ & $<0.001^{* * *}$ \\
\hline
\end{tabular}

${ }^{*} \mathrm{p}<0.05 ; \quad{ }^{* *} \mathrm{p}<0.01 ; \quad * * * \mathrm{p}<0.001$

RED0: reintervention. COPD: Chronic Obstructive Pulmonary Disease IABP: preoperative Intra-Aortic Balloon Pump.. t.Qx: surgery durantion, ICU-LOS: Intensive critical unit length of stay, HOSP-LOS: hospitalization length of stay. Op.Room: Operative Room, ICU: Intensive Critical Unit

\section{DISCUSSION}

Previous studies have reported possible contraindications to early extubation including obesity, female sex, excessive bleeding, inotropic support, use of IABC, hypothermia, prolonged extracorporeal circulation, and prolonged surgery time. ${ }^{17}$. While UFT anesthesia management may not yet be common practice in myocardial revascularization surgery in every practice, published reports have noted that immediate extubation in the Op.Room after cardiac surgery is feasible, safe, and, moreover, has benefits compared to later extubation. ${ }^{2,3,5}$

An aim of this study was to describe the short-term outcome ( $\leq 30$ days) of a consecutive case-series of 673 patients managed by a combination of desflurane, remifentanil, and intercostals nerve block in OPCAB procedures by LAT surgical approach and followed up by multidisciplinary enhance recovery pathway, a "preliminary" protocol including preoperative patient and family educational material, preoperative training and consensus-based standard perioperative management protocol ${ }^{13-15}$ (Appendix 1).

Our patients, a heterogeneous group with varying levels of risk, generally spent a short time in the ICU ( $94.5 \%$ of patients left the ICU within 24 hours) and short Hosp-LOS with relatively low rates of morbidity (2.1\% were readmitted to the ICU) and mortality (1.6\%). In most of our cases, $94.1 .8 \%$ of patients had Hosp-LOS $\leq 4$ days, and followed by a week of at-home nursing care, enabling our patients to return to their daily activities as quickly as possible. We defined as prolonged Hospitalization as length of stay $>4$ days due to the observation of our previous case-series(10); our cut-off matches with others publications(18).

The safety of ICU discharge within the first 24 hours after a CABG has been previously described ${ }^{18}$ and UFT has not been associated with an increased incidence of readmission to the ICU or hospital ${ }^{19}$. In our case-series ,the univariate logistic regression analysis found many factors that influenced to increase the probability 
Perioperative Predictors of Ultra-Fast-Track Failure and Prolonged Hospital Length of Stay in a Preliminary Protocol of Enhanced Recovery after off-Pump Coronary Artery Bypass Grafting

for UFT-failure, including female gender and age $>80$ y.o. Others independent variables were significantly associated with UFT-failure such as EuroSCORE, severity of angina pectoris, previous diagnostic of COPD and COPD severity, poor LV function and PRBC transfusion. While the literature does not suggest that advanced age is a contraindication for UFT anesthesia ${ }^{20}$, female patients and age $>80$ y.o are nevertheless at risk for longer ICU-LOS and Hosp-LOS. However, the strongest risk factors for UFT-failure were revealed by multivariate analysis: Severity of angina pectoris, poor LV function, PRCB transfusion and duration of surgery. In fact, surgery time $>3$ hours increase 70 times the risk of UTF failure, in the same way, transfusion of $\geq 2$ PRCB increase 6 times the risk for UFT-failure and Angina Pectoris grade IV up to 12 times. $\mathrm{EF}<30 \%$ showed a very strong negative influence over success of UTF.

In our study, only 6 patients $(0.9 \%)$ had postoperative neurological dysfunction and stroke, very low stroke rate in comparison with others series of CABG with CBP. On the other hand, OPCAB together with the non-aortic touch technique, especially in the elderly with advanced atherosclerotic disease, avoids the deleterious effects of CPB and reduces the risk for cerebral embolism. ${ }^{21}$ Further, no differences have been found in recall and intraoperative awareness compared with traditional management. ${ }^{22}$

Conventional anesthetic techniques incorporate high doses of opioids such as fentanyl and sufentanil to reduce in traoperative oxidative stress and sympathetic-adrenergic stimulation, those are associated with more than 3-6 hours of mechanical ventilation, require more IV fluids or vasopressors, and require more than 24 hours in the ICU. This prolonged time to emergence delays hospital discharge and could be associated with poorer outcomes. ${ }^{23}$

In our case-serie only 40 patients (5.9\%) had a HospLOS $>4$ days. Majority of the independent variables associated with UFT-failure, also were associated with prolonged hospitalization. Additionally, univariate analysis identifies NYHA functional class, renal failure, Redo and UCI-LOS ( $\mathrm{p}<0.001)$ as risk factors for HospLOS>4days. Multivariate analysis with backward elimination method showed the duration of surgery $(\mathrm{t}-\mathrm{Qx}>3 \mathrm{hrs})(\mathrm{AOR}>75)$ and ICU-LOS $>24 \mathrm{hrs}(\mathrm{AOR}=30)$ $(\mathrm{p}<0.001)$ as the strongest risk factors for prolonged
Hosp-LOS. Otherwise, because the use of elective IABP prevented intra-operative complications in the high-risk patients, it may reduce the probability of prolonged Hosp-LOS, AOR=0.06 [0.01-0.51] $(95 \% \mathrm{CI})$ in this particular group. Elective IABP was used to support and maintain hemodynamic stability during graft construction in our high-risk patients with left main coronary artery disease, unstable angina, recent MI, reoperation and/or EF $\leq 30 \%$. We believe that prophylactic use of IABP may reduce the need for CPB support and emergency sternotomy which could be caused by ischemic events during anastomosis, thus reducing mortality among the patients in our study with the highest risk. ${ }^{30}$ In addition, we used femorofemoral CBP support to keep the heart beating without emergent sternotomy in $0.4 \%$ of our patients

The cornerstone of UFT anesthesia is multimodal perioperative analgesia including regional and local anesthesia based on American Pain Society guidelines. ${ }^{24}$ In our protocol, the use of remifentanil, a short-acting opioid with a very rapid context-sensitive half-life, allows for profound analgesia during surgery without prolonged respiratory depression and with faster emergence. The use of volatile agents (desflurane or sevoflurane) is associated with reduced morbidity and mortality in CABG due to their cardioprotective effects. The choice of an anesthetic regimen based on the administration of these halogenated anesthetics is associated with a better outcome after coronary cardiac surgery. ${ }^{25,26}$ We believe that the combination of desflurane and remifentanil, both of which have cardioprotective effects against ischemic reperfusion injury ${ }^{25-27}$ has also contributed to our outcomes. Extubation in the Op.Room is especially achievable when OPCAB is used, as it decreases operating time and avoids the pathophysiological changes that are generally induced by CPB. ${ }^{8,28}$

TEE is a minimally invasive technique to monitor left ventricular segmental wall motion during OPCAB surgery, but its greatest utility occurs after reperfusion. ${ }^{29}$ Persistent segmental wall motion abnormalities after revascularization could prompt the surgeon to reassess the patency of the coronary bypass graft, which would then reduce the likelihood of postoperative angina pectoris or MI. The rate of postoperative MI in our study was $0.6 \%$.

We believe that avoiding $\mathrm{CPB}$, maintaining 
Perioperative Predictors of Ultra-Fast-Track Failure and Prolonged Hospital Length of Stay in a Preliminary Protocol of Enhanced Recovery after off-Pump Coronary Artery Bypass Grafting

normothermia, using tranexamic acid and maintaining a meticulous surgical technique contributed to an acceptable rate of postoperative bleeding without an important increment of ischemic coronary, stroke, or thrombo-embolic events. Use of the LAT surgical approach could be another factor. Sternotomy is a faster approach, especially in hemodynamic emergencies, and may produce less pain than a traditional posterolateral thoracotomy, however, its association with periosteal reaction and marrow bleeding are concerning.

Although only $16.6 \%$ of our patients required blood transfusions (a result of eschewing CPB and following strict transfusion criteria), Hosp-LOS was nevertheless higher for those patients who received transfusions and were not extubated in the Op.Room ( $p<0.001)$. Our results match Scott et al., ${ }^{9}$ so we found that HospLOS $>4$ days was, in fact, a function of bleeding and needs of transfusions, OR=686 [144.8-3,249] (95\% C.I ). Also, our results matches Zakhary $\mathrm{W}$ et al. ${ }^{31}$ who found similar association between extubation outcome and age, sex, duration of surgery $>3$ hrs.

Our study has limitations, including its descriptive nature and lack of long-term outcome data. In addition, we did not compare our work with those managed by CPB and UFT, neither we compared our results with pre-ERAS series (it is a preliminary protocol that has been recently testing since 2009). According to the literature, we think that standardizing an ERAS protocol for UTF-OPCAB management may be the most reasonable way to obtain the best results ${ }^{15}$, but it must be confirmed by next randomized studies. Nevertheless, for more than 15 years we have used predominantly OPCAB by LAT approach with good results. Unfortunately, we did not include our casesseries before 2010 because with used various types of hypnotics for induction and other volatile agents.

In conclusion, the management described in this study allows very rapid recovery as well as a short ICU and Hosp- LOS, offering the possibility of cost reduction; this fact has permitted us to help a vast number of cardiac patients at our center for years. ${ }^{32}$ In general, age $>80$ y.o, female sex, severity of EuroSCORE and $\geq 2$ PRBCs transfusion were risk factor for UTF-failure and prolonged Hosp-LOS. However, our study has developed two predictive models with high diagnostic validity for UFT-failure and prolonged Hosp-LOS that include as stronger perioperative risk factor transfusion of $\geq 2$ PRCB, severity of angina pectoris, Redo, duration of surgery $>3$ hrs and ICU-LOS $>24 \mathrm{hrs}$ . More multicenter prospective studies with the aim of determining which variables are associated with increased risk for postoperative bleeding, UFT-failure, prolonged ICU-LOS and prolonged Hosp-LOS would enable better management of cardiac surgery patients and perhaps encourage the use of bloodless medicine techniques.

\section{ACKNOWLEDGE}

We would like to thanks Mr. Juan Luis Gómez Martínez ,statistical advisor of St. Halley Statistics for all the support he gave us, statistical guidance and patience.

\section{REFERENCES}

[1] Zayat R, Menon AK, Goetzenich A, et al. Benefits of ultra-fast-track anesthesia in left ventricular assist device implantation: a retrospective, propensity score matched cohort study of a fouryear single center experience. J Cardiothorac Surg. 2017;12(1):10.

[2] Borracci RA, Dayan R, Rubio M, Axelrud G, Ochoa G, Rodriguez LD. [Operating room extubation (ultra fast-track anesthesia) in patients undergoing on-pump and off-pump cardiac surgery]. Arch Cardiol Mex. 2006;76(4):383-389.

[3] Lee TW, Jacobsohn E. Pro: tracheal extubation should occur routinely in the operating room after cardiac surgery. J Cardiothorac Vasc Anesth. 2000;14(5):603-610.

[4] Maddali MM, Al-Jadidi AM, Zacharias S. Novel anaesthetic approach for surgical access and haemodynamic management during offpump coronary artery bypass through a left thoracotomy. Indian J Anaesth. 2012;56(1):75-78.

[5] Straka Z, Brucek P, Vanek T, Votava J, Widimsky P. Routine immediate extubation for off-pump coronary artery bypass grafting without thoracic epidural analgesia. Ann Thorac Surg. 2002;74(5):1544-1547.

[6] Cheng DC, Karski J, Peniston C, et al. Morbidity outcome in early versus conventional tracheal extubation after coronary artery bypass grafting: a prospective randomized controlled trial. J Thorac Cardiovasc Surg. 1996;112(3):755-764. 
Perioperative Predictors of Ultra-Fast-Track Failure and Prolonged Hospital Length of Stay in a Preliminary Protocol of Enhanced Recovery after off-Pump Coronary Artery Bypass Grafting

[7] Flynn M, Reddy S, Shepherd W, et al. Fast-tracking revisited: routine cardiac surgical patients need minimal intensive care. Eur J Cardiothorac Surg. 2004;25(1):116-122.

[8] Sellke FW, Chu LM, Cohn WE. Current state of surgical myocardial revascularization. Circ J. 2010;74(6):1031-1037.

[9] Scott BH, Seifert FC, Grimson R, Glass PS. Resource utilization in on- and off-pump coronary artery surgery: factors influencing postoperative length of stay--an experience of 1,746 consecutive patients undergoing fast-track cardiac anesthesia. J Cardiothorac Vasc Anesth. 2005;19(1):26-31.

[10] Guida MC, Pecora G, Bacalao A, Munoz G, Mendoza $\mathrm{P}$, Rodriguez L. Multivessel revascularization on the beating heart by anterolateral left thoracotomy. Ann Thorac Surg. 2006;81(6):21422146 .

[11] Xiao LB, Zhang YH, Zhou JW, et al. The clinical research of off-pump coronary artery bypass grafting by small incision at the left chest. Eur Rev Med Pharmacol Sci. 2016;20(2):305-310.

[12] Zhang L, Cui Z, Song Z, et al. Minimally invasive direct coronary artery bypass for left anterior descending artery revascularization - analysis of 300 cases. Wideochir Inne Tech Maloinwazyjne. 2015;10(4):548-554.doi:10.5114/ wiitm.2015.55842

[13] Ljungqvist O, Scott M,Fearon K.Enhanced Recovery after Surgery. JAMA Surg 2017;11:E1E7.doi:10.1001/jamasurg.2016.4952.

[14] Nanavati AJ,Nagral S,Prabhakar S. Fast Track Surg in India.Natl Med J India 2014;27:79-83.

[15] Mandani A, Fiore J.F, Wang Y, Bejjani J, Sivakumaran L, Mata J,Watson D, Carli F, Mulder D, Sirois Ch, Ferri L, Feldman L. An enhanced recovery pathway reduce duration of stay and complications after open pulmonary lobectomy. Surgery 2015;158: 899-910.

[16] Gaies MG, Gurney JG, Yen AH, et al. Vasoactiveinotropic score as a predictor of morbidity and mortality in infants after cardiopulmonary bypass. Pediatr Crit Care Med. 2010;11(2):234-238.
[17] Bucerius J, GummertJF, Walther T, etal. Predictors of prolonged ICU stay after on-pump versus offpump coronary artery bypass grafting. Intensive Care Med. 2004;30(1):88-95.

[18] Calafiore AM, Scipioni G, Teodori G, et al. Day 0 intensive care unit discharge - risk or benefit for the patient who undergoes myocardial revascularization? Eur J Cardiothorac Surg. 2002;21(3):377-384.

[19] Celkan MA, Ustunsoy H, Daglar B, Kazaz H, Kocoglu H. Readmission and mortality in patients undergoing off-pump coronary artery bypass surgery with fast-track recovery protocol. Heart Vessels. 2005;20(6):251-255.

[20] London MJ, Shroyer AL, Coll JR, et al. Early extubation following cardiac surgery in a veterans population. Anesthesiology. 1998;88(6):14471458.

[21] Mishra YK, Mishra M, Malhotra R, Meharwal ZS, Kohli V, Trehan N. Evolution of Off-Pump Coronary Artery Bypass Grafting over 15 Years: A Single-Institution Experience of 14,030 Cases. Innovations (Phila). 2005;1(2):88-91.

[22] Vance JL, Shanks AM, Woodrum DT. Intraoperative bispectral index monitoring and time to extubation after cardiac surgery: secondary analysis of a randomized controlled trial. $B M C$ Anesthesiol. 2014;14:79.

[23] Lison S, Schill M, Conzen P. Fast-track cardiac anesthesia: efficacy and safety of remifentanil versus sufentanil. J Cardiothorac Vasc Anesth. 2007;21(1):35-40.

[24] Chou R, Gordon DB, de Leon-Casasola OA, et al. Management of Postoperative Pain: A Clinical Practice Guideline From the American Pain Society, the American Society of Regional Anesthesia and Pain Medicine, and the American Society of Anesthesiologists' Committee on Regional Anesthesia, Executive Committee, and Administrative Council. J Pain. 2016;17(2):131-157.

[25] Landoni G, Biondi-Zoccai GG, Zangrillo A, et al. Desflurane and sevoflurane in cardiac surgery: a meta-analysis of randomized clinical trials. $J$ Cardiothorac Vasc Anesth. 2007;21(4):502-511. 
Perioperative Predictors of Ultra-Fast-Track Failure and Prolonged Hospital Length of Stay in a Preliminary Protocol of Enhanced Recovery after off-Pump Coronary Artery Bypass Grafting

[26] Meco M, Cirri S, Gallazzi C, Magnani G, Cosseta D. Desflurane preconditioning in coronary artery bypass graft surgery: a double-blinded, randomised and placebo-controlled study. Eur $J$ Cardiothorac Surg. 2007;32(2):319-325.

[27] Greco M, Landoni G, Biondi-Zoccai G, et al. Remifentanil in cardiac surgery: a meta-analysis of randomized controlled trials. J Cardiothorac Vasc Anesth. 2012;26(1):110-116.

[28] Tempe DK, Gandhi A, Virmani S. Resource utilization in on- and off-pump coronary artery surgery: factors influencing postoperative length of stay--an experience of 1,746 consecutive patients undergoing fast-trackcardiacanesthesia. J Cardiothorac Vasc Anesth. 2006;20(1):128; author reply 128-129.

[29] Kapoor PM, Chowdhury U, Mandal B, Kiran U, Karnatak R. Trans-esophageal echocardiography in off-pump coronary artery bypass grafting. Ann Card Anaesth. 2009;12(2):167.

[30] Sa MP, Ferraz PE, Escobar RR, et al. Prophylactic intra-aortic balloon pump in high-risk patients undergoing coronary artery bypass surgery: a meta-analysis of randomized controlled trials. Coron Artery Dis. 2012;23(7):480-486.

[31] Zakhary Waseem,Lindner Jacob,Sgouropoulou Sophia,Eibel Sarah,Prost Stefan,Scholz Markus,Ender Joerg.Independent Risk factors for Fast.Track protocol in preselected cardiac surgery patients..J.Cardithoracic and Vascular Anesth.2017;31(3):1150

[32] Quigley RL, Reitknecht FL. A coronary artery bypass "fast-track" protocol is practical and realistic in a rural environment. Ann Thorac Surg. 1997;64(3):706-709.

\section{AbBreviations}

OCAPB: off-pump coronary artery bypass, LAT: left anterolateral thoracotomy, CABG: on- pump coronary artery bypass graft, UFT: Ultra- fast-track, UTF-success: extubation in operative room, UFTfailure: extubation in intensive critical unit ,NYHA: New York heart association, ASA: American Society of Anaesthesiologist, Op.Room: operatory room, ICU: intensive critical unit, ICU-LOS: intensive critical unit length of stay,Hosp-LOS: hospitalization length of stay
,ERAS: enhance recovery after surgery, PRCB: packet of red cell blood, EF: ejection fraction, OR: Odds ratio, AOR: Adjusted Odds ratio, CI: confidence interval ,AUC: area under curve, t-Qx: duration of surgery , IBP: invasive blood pressure, $\mathbf{P R}$ : pulse rate, RR: respiratory rate, TOF: tren of four, NSAIDS: non steroidal antiinflammatory drugs, CPB: cardiopulmonary bypass, IABP: intra-aortic ballom pump, Redo: previous CABG, ARDS: respiratory distress syndrome, COPD: Chronic Obstructive pulmonary disease, BMI: Body mass index, DM: diabetes mellitus, HTA: arterial hypertension, PAD: Peripheral artery disease, TEE: Trans-esophageal echocardiography, VAS: Visual analogue scale, MI: myocardial infarct, RWMA: regional wall motion abnormality, A.P(CCS): angina pectoris Canadian Cardiovascular Society grades, LV: Left Ventricular, CAD: coronary arterial disease, AF: Atrial fibrillation, PCI: percutaneus coronay inteventional procedure, LIMA: Left internal mammary artery. RIMA: right internal mammary artery.

Appendix 1. GOALS OF ENHANCED RECOVERY AFTER OPCAB-ALT PROTOCOL

\section{Pre-Operative}

- Standardized patient and family education (minimum 2 interviews): complete information about protocol, advice regarding respiratory exercise, early mobilization, smoking and alcohol cessation.

- Information booklet with daily goals. Including red flag at home (postoperative warning signs)

- Pre-anesthesia evaluation and take an informed consent

- Optimization of any pre-existing co-morbidity

- Minimal starvation (8hrs for solid and $2 \mathrm{hrs}$ for clear liquids*)

- Anesthesia premedication plus $100 \mathrm{ml}$ oral carbohydrate drink* 2 hrs before surgery.

- Preoperative antibiotic prophylaxis (cefazolina $2 \mathrm{gr}$,or Vancomycin 1gr if Beta-lactamic allergy

\section{Operative}

- UFT anesthetic protocol (DesfluraneRemifentanil)

- Pre-incisional Intercostals Block with bupivacaine 
Perioperative Predictors of Ultra-Fast-Track Failure and Prolonged Hospital Length of Stay in a Preliminary Protocol of Enhanced Recovery after off-Pump Coronary Artery Bypass Grafting

- Intercostals(paravertebral) catheter for fluid diet on 6-8hrs, if tolerate Ensure Clearß.1 $1^{\text {st }}$ postoperative analgesia P.O day diet as tolerate following nutrition

- Goal directed fluid and blood transfusion therapy

- $\quad$ Avoid hypothermia(active warming).

- Temporal use of nasogastric tubes. PONV prophylaxis

- Alveolar recapture maneuvers

\section{POST-OPERATIVE}

- Multimodal analgesia plus intercostals local anesthesia by catheter(remove $48 \mathrm{hrs}$ followed by tramadol drops as rescue)

- $\quad$ Chest physiotherapy every 4 hours

- $\quad$ Chest tubes: (ICU) at suction $-20 \mathrm{cmH} 20.1^{\text {st }} \mathrm{P} .0$ day: stop suction. $2^{\text {nd }}$ P.O day remove tube $\mathrm{N}^{\circ} 1$ if $<200 \mathrm{ml} / 24 \mathrm{~h}$. $3^{\text {th }}$ remove tube $\mathrm{N}^{\circ} 2$ if $<200 \mathrm{ml} / 24$ $\mathrm{h}$ nonchylous and no air leak

- $\quad$ Arterial line removed at ICU discharge. Central line removed $24 \mathrm{hr}$ before hospital discharge.

- Urinary drain removed at ICU discharge if adequate urine output

- Early nutrition: No nasogastric tube. ICU clear services advices. PONV prophylaxis, lactulose $15 \mathrm{ml} /$ day if necessary.

- Thromboembolism Prophylaxis with lowmolecular weight heparine, following ICU protocol.

- Early mobilization: ICU passive physiotherapy. $1^{\text {st }} \mathrm{P} .0$ day up in chair with assistance as tolerate. $2^{\text {nd }}$ PO day up in chair 3-4 times/day for 45 minutes and ambulate in hallway 1 time/day with assistance for 20 minutes. 3th PO day: out of bed for meals and minimum 4 hours during the day, walking with assistance 4 times/day for 20 minutes. From $4^{\text {th }}$ PO day onwards increase ambulation to 30 minutes 4-5 times/day and 6-8 hours out bed.

\section{POST-DischaRgE}

- Ensure 30 days follow-up including: ERAS nurses performs daily at-home wound care for a week. Phone call at 48 hour by surgeon ERAS coordinator. Permanent phone call contact and any emergency visit. Followed-up at 1, 2, 4, 6, and 8 weeks postoperatively

Citation: Carlos Ramirez-Paesano, MD, Gustavo Muñoz-Galarce, MD et al. Perioperative Predictors of Ultra-Fast-Track Failure and Prolonged Hospital Length of Stay in a Preliminary Protocol of Enhanced Recovery after off-Pump Coronary Artery Bypass Grafting. Archives of Anesthesiology. 2018; 1(2): 10-27.

Copyright: (C) 2018 Carlos Ramirez-Paesano, MD, Gustavo Muñoz-Galarce, MD et al. This is an open access article distributed under the Creative Commons Attribution License, which permits unrestricted use, distribution, and reproduction in any medium, provided the original work is properly cited. 